\title{
Efficient Hyperpolarization of U-13 C-Glucose Using Narrow-Line UV-Generated Labile
} Free Radicals

Capozzi, Andrea; Patel, Saket; Gunnarsson, Christine Pepke; Marco-Rius, Irene; Comment, Arnaud; Karlsson, Magnus; Lerche, Mathilde Hauge; Ouari, Olivier; Ardenkjær-Larsen, Jan Henrik

Published in:
ANGEWANDTE CHEMIE

Link to article, DOI:

10.1002/anie.201810522

Publication date:

2018

Document Version

Peer reviewed version

Link back to DTU Orbit

Citation $(A P A)$ :

Capozzi, A., Patel, S., Gunnarsson, C. P., Marco-Rius, I., Comment, A., Karlsson, M., Lerche, M. H., Ouari, O., \& Ardenkjær-Larsen, J. H. (2018). Efficient Hyperpolarization of U-13 C-Glucose Using Narrow-Line UV-

Generated Labile Free Radicals. ANGEWANDTE CHEMIE, 57, 1 - 6. https://doi.org/10.1002/anie.201810522

\section{General rights}

Copyright and moral rights for the publications made accessible in the public portal are retained by the authors and/or other copyright owners and it is a condition of accessing publications that users recognise and abide by the legal requirements associated with these rights.

- Users may download and print one copy of any publication from the public portal for the purpose of private study or research.

- You may not further distribute the material or use it for any profit-making activity or commercial gain

- You may freely distribute the URL identifying the publication in the public portal 


\title{
Efficient Hyperpolarization of U-13C-Glucose using Narrow-line UV-generated Labile Free Radicals
}

\author{
Andrea Capozzi, ${ }^{* \#[a]}$ Saket Patel, ${ }^{\#[b]}$ Christine Pepke Gunnarsson, ${ }^{[a]}$ Irene Marco-Rius, ${ }^{[c]}$ Arnaud \\ Comment, ${ }^{[c, d]}$ Magnus Karlsson, ${ }^{[a]}$ Mathilde H. Lerche, ${ }^{[a]}$ Olivier Ouari, ${ }^{[b]}$ and Jan Henrik Ardenkjær- \\ Larsen $^{[a]}$
}

\begin{abstract}
Free radicals generated via irradiation with UV-light of a frozen solution containing a fraction of pyruvic acid (PA), have demonstrated their dissolution Dynamic Nuclear Polarization (dDNP) potential providing up to $30 \%\left[1-{ }^{13} \mathrm{C}\right] \mathrm{PA}$ liquid-state polarization. Moreover, their labile nature has proven to pave a way to nuclear polarization storage and transport. Herein, differently from the case of $\mathrm{PA}$, we tackled the issue of providing dDNP UV-radical precursors, trimethylpyruvic acid (TriPA) and its methyl-deuterated form $d_{9}$-TriPA, not involved in any metabolic pathway. The ${ }^{13} \mathrm{C}$ dDNP performance was evaluated for hyperpolarization of $\left[\mathrm{U}-{ }^{13} \mathrm{C}_{6}, 1,2,3,4,5,6,6-\mathrm{d}_{7}\right]-\mathrm{D}$ glucose. The generated UV-radical proved to be a versatile and highly efficient polarizing agent providing, after dissolution and transfer (10 s), $a{ }^{13} \mathrm{C}$ liquid-state polarization up to $32 \%$.
\end{abstract}

During the last decade, ${ }^{13} \mathrm{C}$ hyperpolarized (HP) magnetic resonance imaging (MRI) and spectroscopy (MRS) have encountered a tremendous development, showing convincing demonstrations in detecting and monitoring biochemical changes in real time in both clinical and preclinical studies. ${ }^{[1]}$ Among the different hyperpolarization techniques used to increase the NMR sensitivity of the substrate, dissolution Dynamic Nuclear Polarization (dDNP) is the most widespread one, because of its versatility in biomedical applications. ${ }^{[2]}$ In particular, hyperpolarization of ${ }^{13} \mathrm{C}$ enriched molecules shows a combination of features that make it the ideal nucleus for real-time metabolic studies: ${ }^{[3]}$ ubiquitous presence in biomolecules, large chemical

*[a] Dr A. Capozzi, Dr M. Karlsson, MSc C. P. Gunnarsson, Dr M. H. Lerche, Prof J. H. Ardenkjær-Larsen

Center for Hyperpolarization in Magnetic Resonance, Department of Electrical Engineering

Technical University of Denmark, Building 349, 2800 Kgs Lyngby (Denmark)

E-mail: andcapo@electro.dtu.dk

Homepage: www.hypermag.dtu.dk

[b] MSc S. Patel, Dr O. Ouari

Institut de Chimie Radicalire

Aix-Marseille Université, CNRS, ICR UMR 7273, 13397 Marseille Cedex 20 (France)

[c] Dr I. Marco-Rius, Dr. A. Comment Cancer Research UK Cambridge Institute University of Cambridge, Li Ka Shing Centre, Cambridge (United Kingdom)

[d] $\operatorname{Dr}$ A. Comment

General Electric Healthcare, Chalfont St Giles, Buckinghamshire HP8 4SP (United Kingdom) shift dispersion (possibility to easily distinguish between several substrates), low natural abundance (absence of a background signal) and relatively long nuclear longitudinal relaxation time $\left(T_{1 n}\right)$ at specific molecular positions (e.g. carbonyl groups, tens of seconds). Indeed in a dDNP experiment, enhancement of the nuclear polarization (i.e. the relative difference between the populations of the two spin eigenstates) takes place ex-situ in the so-called DNP polarizer. The latter provides a moderate magnetic field $(3.35-7 \mathrm{~T})$ and a cold environment $(1-1.5 \mathrm{~K})$ hosting a glassy frozen sample that includes the substrate of interest (at molar concentration) and unpaired electrons (15 - $50 \mathrm{mM}$ ), in the form of organic free radicals. After the microwaves driven polarization transfer from the electron spins to the surrounding nuclear spins reaches a steady-state, the frozen sample is quickly dissolved in a hot buffer and ejected from the polarizer. ${ }^{[2 b]}$ Extracting the sample in the liquid state is at the same time the strength and weakness of the technique. On the one hand, the polarization created in the solid state is preserved, generating a high sensitivity metabolic probe ready to be injected. But on the other, a dedicated polarizer is needed as close as possible to the MRI scanner, since the HP state is $T_{1 n}$-limited. A way to circumvent this limitation is to employ labile radicals.

Labile radicals, generated via irradiation with UV-light of a frozen solution containing a fraction of PA (UV-PA), have demonstrated their dDNP capability on PA itself and other substrates, ${ }^{[4]}$ providing up to $30 \%\left[1-{ }^{13} \mathrm{C}\right] \mathrm{PA}$ liquid-state polarization at optimal conditions. ${ }^{[5]}$ Moreover, their unique feature to recombine around $190 \mathrm{~K}$ allows recovery of a HP solution without radicals and has proven to pave the way, together with other approaches, ${ }^{[6]}$ to nuclear polarization storage and transport. Indeed, quenching the radicals when the sample is still frozen gives the possibility of extracting it from the DNP polarizer and dissolve the HP sample far away from its production site. ${ }^{[7]}$

In the present work we tackled the issue of providing dDNP UVradical precursors not involved in any metabolic pathway and producing labile radicals with improved ${ }^{13} \mathrm{C}$ dDNP performance compared to UV-PA. To this end, we investigated the photochemical properties of two a-keto acids, trimethylpyruvic acid (TriPA) and its methyl-deuterated form dg-TriPA (see Figure 1). We studied their dDNP efficiency as non-persistent radicals on isotope labelled $D$-glucose $\left({ }^{13} C_{6}, 1,2,3,4,5,6,6-d_{7}\right)$, a substrate showing increasing interest in the hyperpolarization community thanks to the richer metabolic pathways it can give access to, compared to the routinely used $\left[1-{ }^{13} \mathrm{C}\right]$ pyruvate. ${ }^{[8]}$

Narrow ESR-line radicals (e.g. Trityls or BDPA), are well known to provide good direct DNP enhancements on ${ }^{13} \mathrm{C}$ nuclei. ${ }^{[9]}$ Although UV-PA has a thinner spectrum than the well-known broad nitroxyl radicals (e.g. TEMPO and TEMPOL), it still shows an ESR-line too broad compared to the ${ }^{13} \mathrm{C}$ Larmor frequency, providing efficient DNP on protons. ${ }^{[4 a]}$ Thus, it may not be the optimal precursor for direct ${ }^{13} \mathrm{C}$ nuclei polarization in general. 


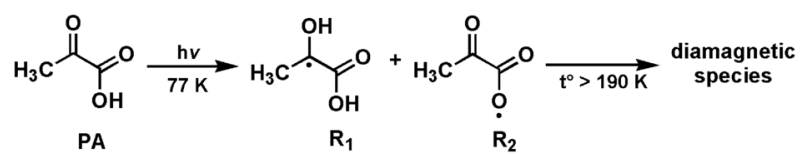<smiles>CC(C)(C)C(=O)C(=O)OC(=O)C(=O)OC(=O)C(=O)O</smiles>

Figure 1. Free radicals generated by UV-irradiation of PA and structures of TriPA and d9-TriPA.

The generation of labile paramagnetic species from UV-light illumination of $\alpha$-keto acids has been explained by photoexcitation of the $n-\pi^{*}$ transition $(300-350 \mathrm{~nm})$ of the $\alpha$-carbonyl group, followed by efficient intersystem crossing (ISC) to an excited triplet state $\left({ }^{3} \mathrm{PA}^{*}\right) .{ }^{[10]}$ When $\mathrm{PA}$ is the photoactive precursor, ${ }^{3} \mathrm{PA}^{*}$ can react with another $\mathrm{PA}$ molecule and two paramagnetic intermediates are expected to appear: the ketyl radical $\mathrm{CH} 3 \mathrm{C}(\mathrm{OH}) \mathrm{C}(\mathrm{O}) \mathrm{OH}$ (R1 in Figure 1) and the acyloxyl radical $\mathrm{CH} 3 \mathrm{C}(\mathrm{O}) \mathrm{C}(\mathrm{O}) \mathrm{O}$ ( $\mathrm{R} 2$ in Figure 1); the latter can then decarboxylates into the acetyl radical $\mathrm{CH} 3 \dot{\mathrm{C} C}(\mathrm{O}) .{ }^{[11]}$ These radicals are extremely unstable at room temperature, but can be, at least partially, "captured" when the UV-irradiation takes place in liquid nitrogen (77 K). ${ }^{[12]}$ Having a clear understanding of which intermediates survive is crucial for the appropriate choice or chemical synthesis of UV-radical precursors with improved ${ }^{13} \mathrm{C}$ dDNP properties. To clarify this point UV-irradiation was performed in liquid nitrogen on frozen solutions of glycerol:water $50: 50(\mathrm{v} / \mathrm{v})(\mathrm{GW} 55)$ containing $10 \%$ of natural abundance PA or $\mathrm{PA}$ with site specific ${ }^{13} \mathrm{C}$ labelling.
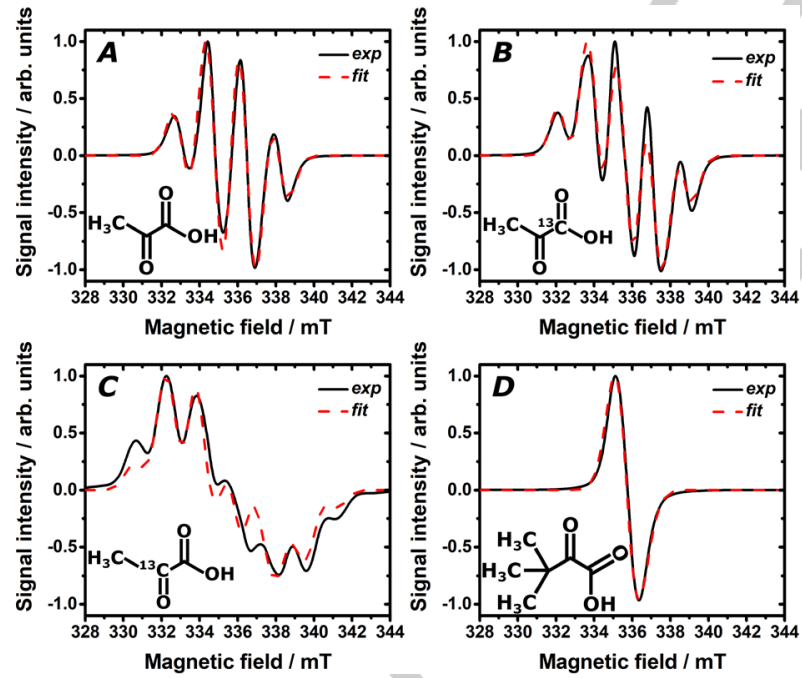

Figure 2. X-band ESR measurement (black line) and ESR spectrum fit (dashed red line) after 5 min UV-light irradiation at $77 \mathrm{~K}$ of a single $4.0 \pm 0.2 \mu \mathrm{L}$ bead of a frozen solution containing PA:GW55 1:9 (v/v)(A); [1-13 C]PA:GW55 1:9 (v/v) (B); $\left[2-{ }^{13} \mathrm{C}\right] P A: G W 55$ 1:9 (v/v) (C); TriPA:GW55 1:9 (v/v) (D). GW55 is an abbreviation for the solvent composition glycerol:water 50:50 (v/v). In the insert the structural formula of the radical precursor is shown.

In Figure 2A to 2C, the X-band ESR spectrum measured at $77 \mathrm{~K}$ (black line) and the corresponding fit (dashed red line) are reported for UV-irradiated PA:GW55 1:9 (v/v), [1-13C]PA:GW55 $1: 9(\mathrm{v} / \mathrm{v})$ and $\left[2-{ }^{13} \mathrm{C}\right] P A: G W 551: 9(\mathrm{v} / \mathrm{v})$, respectively. While the same g-tensor $=\left[\begin{array}{lll}2.0036 & 2.0027 & 2.0007\end{array}\right]$ could be used for fitting all three spectra, it is interesting to see how the hyperfine coupling changes as a function of the ${ }^{13} \mathrm{C}$ labelling of the PA molecule. In panel $A$ the peak quartet spectrum was reproduced through an isotropic coupling $a_{1 H}=1.67 \mathrm{mT}$ to the magnetically equivalent methyl protons, in good agreement with previous studies. ${ }^{[12]}$ In panel $\mathrm{B}$ the ${ }^{13} \mathrm{C}$ labelling in $\mathrm{C} 1$ position generated a peak quintet and it was taken into account by adding an extra isotropic coupling $a_{13 \mathrm{C}}=1.07 \mathrm{mT}$. In panel $\mathrm{C}$ the strong $\left(a_{13 \mathrm{C}}=2.82 \mathrm{mT}\right)$ coupling to the ${ }^{13} \mathrm{C}$ nucleus in $\mathrm{C} 2$ position changed dramatically the ESR spectrum appearance generating almost a doublet of quartet (for more details about spectra fitting see Supporting Information). Thus, ${ }^{13} \mathrm{C}$ labelling affected the spectrum in both cases with a stronger effect on $\mathrm{C} 2$. On the basis of the foregoing discussion, we can state that the radical stabilized by the cold environment and thus, active DNP wise is the ketyl one. It appears clear now that the choice of TriPA as UV-radical precursor provides a more isolated electron environment: by pushing the methyl protons two carbon positions away, the hyperfine coupling was reduced $\left(a_{1 H}\right.$ $=0.11 \mathrm{mT}$ ) generating a sharp single line X-band ESR spectrum (see Figure 2D). Deuteration of the methyl groups decreased the hyperfine coupling $\left(\mathrm{a}_{2 \mathrm{H}}=0.02 \mathrm{mT}\right)$ and the ESR linewidth even further (data not shown). Moreover, the g-anisotropy was less pronounced when TriPA and $\mathrm{d}_{9}$-TriPA were the radical precursors: g-tensor $=\left[\begin{array}{lll}2.0031 & 2.0022 & 2.0012\end{array}\right]$ (see Supporting Information for spectra comparison at 6.7 T).
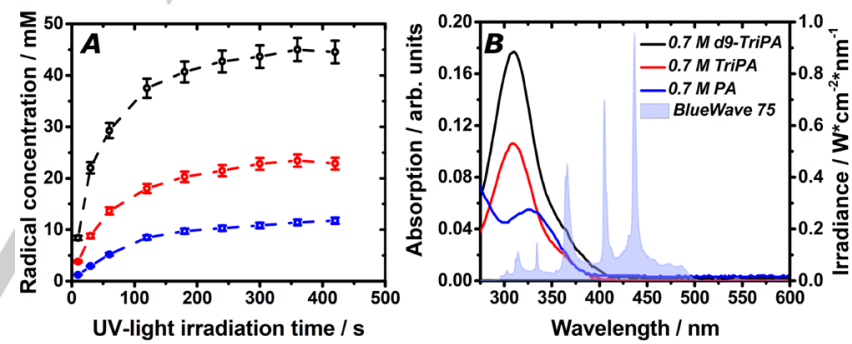

Figure 3. Radical generation at $77 \mathrm{~K}$ as a function of UV-irradiation time for frozen mixtures of $0.7 \mathrm{M}$ TriPA (red curve), dg-TriPA (black curve) and PA (blue curve) with $2 \mathrm{M}\left[\mathrm{U}-{ }^{13} \mathrm{C}, \mathrm{d}_{7}\right]-\mathrm{D}$-glucose dissolved in GW55. Measurements were performed on a single $4.0 \pm 0.2 \mu \mathrm{L}$ frozen bead immersed in liquid nitrogen (A). Room temperature UV-vis absorption measurements for the same sample solutions are shown in (B) (left y-scale); the light blue coloured area represents the spectral irradiance (right y-scale) of the UV-source (Dymax, BlueWave 75) at maximum power $\left(19 \mathrm{~W} / \mathrm{cm}^{2}\right)$ used to photo-generate the radicals.

As previously described, ${ }^{[5]}$ also UV-irradiated dDNP samples were prepared in liquid nitrogen outside from the polarizer. We studied frozen solutions consisting of $0.7 \mathrm{M}$ TriPA and $\mathrm{d}_{9}$-TriPA with $2 \mathrm{M}\left[\mathrm{U}-{ }^{13} \mathrm{C}, \mathrm{d}_{7}\right]$ glucose dissolved in GW55. From now onward the two preparations will be addressed as TriPA_DNP-sample and $d 9$-TriPA_DNP-sample, respectively. Aiming at minimizing as much as possible the precursor molecules amount in the sample, the chosen concentration (corresponding to $10 \%$ of the final volume) was the lowest providing enough radical to perform efficient DNP at $6.7 \mathrm{~T}$ and $1.1 \mathrm{~K}$ for both samples (see Supporting Information). In Figure $3 \mathrm{~A}$ the radical generation as a function of UV-irradiation time is reported. In both cases irradiating the 
sample for $300 \mathrm{~s}$ was sufficient to reach the radical concentration plateau. Surprisingly the radical yield was doubled when $d_{9}$-TriPA was employed. For the sake of comparison a third sample containing $0.7 \mathrm{M}$ of PA was prepared. The latter generated half of the radical concentration obtained with the TriPA sample. We found experimental evidence that the TriPA light absorption in correspondence to the $n-\pi^{*}$ transition was approximately half as strong as $d_{9}$-TriPA and twice as strong as PA (Figure 3B). This observation together with the fact that, for a given precursor concentration, the radical yield is strictly UV-light power dependent ${ }^{[5]}$ may explain the behaviour reported in Figure $3 \mathrm{~A}$. Another possible explanation, or confounding factor, could be related to the different amount of precursor in the hydrate form. Indeed, when in water solution, hydration of the carbonyl group of ketones is a well-known phenomenon. ${ }^{[11]}$ The hydrate form of the precursor molecule is not photoactive and does not contribute to the generation of any radical. ${ }^{[13]}$ We estimated the amount of trimethylpyruvate, $d_{9}$-trimethylpyruvate and pyruvate hydrate in the three liquid mixtures via ${ }^{13} \mathrm{C}$ NMR. A higher amount of hydrate characterized the sample containing PA, but no significant difference in hydrate amounts was observed between the other two (Supporting Information). Although beyond the scope of the present work, the physical chemistry behind the origin of the isotopic effect characterizing $\mathrm{d}_{9}$-TriPA remains unclear.

In order to obtain efficient DNP, it is important to achieve a homogenous radical distribution inside the frozen sample. This is a minor issue when using chemical doping, ${ }^{[14]}$ but may be more challenging when radicals are photo-induced. Using a methodology previously described, ${ }^{[5]}$ we demonstrated that for this low concentration of precursor the paramagnetic centres were homogeneously induced inside the sample volume (see Supporting Information).
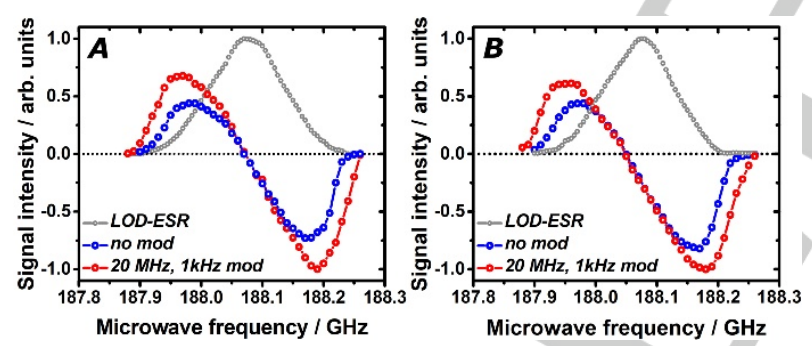

Figure 4. The longitudinal detected (LOD) ESR spectrum (grey curve) and the ${ }^{13} \mathrm{C}$ DNP microwaves sweep without modulation (blue curve) and with modulation (red curve) are reported for TriPA DNP-sample (panel A) and $d_{9}$ TriPA_DNP-sample (panel B). All measurements were performed at $6.7 \mathrm{~T}$ and $1.1 \mathrm{~K}$. Samples were UV-irradiated in liquid nitrogen for $300 \mathrm{~s}$.

In Figure 4 the ESR spectrum and ${ }^{13} \mathrm{C}$ DNP microwaves sweep measured at $6.7 \mathrm{~T}$ and $1.1 \mathrm{~K}$ are shown for TriPA_DNP-sample and d9-TriPA_DNP-sample. Even at high field, where the anisotropic Zeeman interaction dominates the linewidth, ${ }^{[15]}$ the deuterated sample showed a slightly narrower ESR spectrum (grey curves) because of the reduced hyperfine coupling to the methyl groups (numerical values are reported in Table 1). Nevertheless this feature did not generate any significant difference between the widths of the two ${ }^{13} \mathrm{C}$ DNP sweeps (blue curves). For both samples the maximum positive and negative DNP enhancement (DNP+ and DNP-) appeared at $187.98 \mathrm{GHz}$ and $188.17 \mathrm{GHz}$, respectively, with $|\mathrm{DNP}-|>|\mathrm{DNP}+|$. Modulation of the microwaves frequency improved the DNP enhancement (red curve). A stronger effect was observed on the protonated sample: at optimal conditions $(20 \mathrm{MHz}$ of modulation amplitude, $1 \mathrm{kHz}$ of modulation frequency), the DNP- value improved by $37 \%$ for TriPA_DNP-sample and $23 \%$ for $d_{9}$ TriPA_DNP-sample. This difference is in good agreement with the different radical concentration generated in the two samples. Indeed, a higher radical concentration yields stronger electron spin dipolar coupling. The latter, especially at high field, represents a key parameter to efficiently saturate the radical ESR line and provide better DNP performance, thus decreasing the microwaves modulation effect. ${ }^{[16]}$

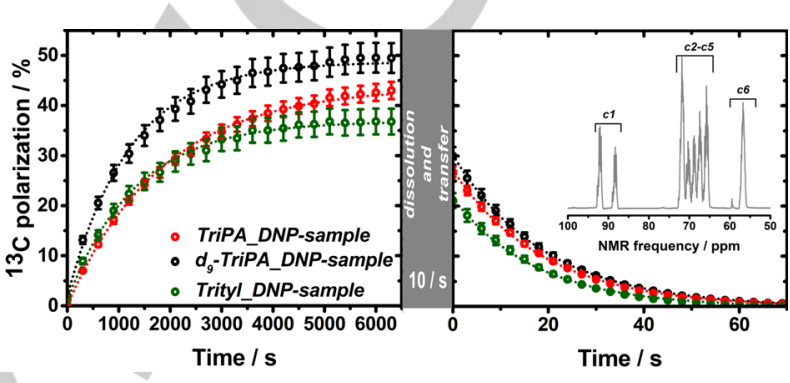

Figure 5. The ${ }^{13} \mathrm{C}$ polarization solid-state build-up (left panel) and liquid-state relaxation (right panel) are showed for TriPA_DNP-sample, $d_{9}$-TriPA_DNPsample and Trityl_DNP-sample. Solid-state measurements were performed at 6.7 $\mathrm{T}$ and $1.1 \mathrm{~K}$ by $\mathrm{mw}$ irradiation at $188.19 \mathrm{GHz}(20 \mathrm{MHz}$ amplitude modulation and $1 \mathrm{kHz}$ frequency modulation) for TriPA_DNP-sample and $d_{9}$-TriPA_DNPsample; Trityl_DNP-sample was polarized at the $187.94 \mathrm{GHz}$ (no microwaves modulation) corresponding to the optimum for Trityl (see Supporting Information). Liquid-state measurements were acquired on 9.4 Thigh resolution magnet equipped with a $5 \mathrm{~mm}$ NMR probe kept at $40^{\circ} \mathrm{C}$. The transfer of the HP solution from the polarizer to the high resolution magnet took $10 \mathrm{~s}$. In the inset the HP $\left[\mathrm{U}-{ }^{13} \mathrm{C}, \mathrm{d}_{7}\right]-\mathrm{D}$-glucose NMR spectrum corresponding to the first point of $d_{9}$-TriPA_DNP-sample signal decay is reported (C2-C5 DNP enhancement = 41000).

TriPA_DNP-sample and dg-TriPA_DNP-sample were polarized at optimal conditions (microwaves frequency $188.19 \mathrm{GHz}$ with \pm 20 $\mathrm{MHz}$ frequency modulation at a rate of $1 \mathrm{kHz}$ ) to estimate the maximum achievable DNP enhancement. The build-up time constants $\left(T_{b}\right)$ were $1836 \pm 28 \mathrm{~s}$ and $1230 \pm 30 \mathrm{~s}$, respectively $(\mathrm{n}=3)$. Once at the plateau the samples were quickly dissolved and transferred ( $\left.T_{\text {trans }}=10 \mathrm{~s}\right)$ to a $9.4 \mathrm{~T}$ high resolution magnet kept at $40{ }^{\circ} \mathrm{C}$ (see Figure 5). The ${ }^{13} \mathrm{C}$ liquid-state polarization $\left(P_{L S}\right)$ was $26.8 \pm 1.1 \%$ for TriPA_DNP-sample and $30.1 \pm 1.8 \%$ for $d g$ TriPA_DNP-sample; in both cases the liquid state $T_{1 n}$ was close to $20 \mathrm{~s}(\mathrm{n}=3)$. Because of the excessively long spin-lattice relaxation time in the solid state $(>13 \mathrm{~h})$, the polarization value at the moment of dissolution $\left(P_{S S}\right)$ was measured only once for each sample. The result was in good agreement with the value back calculated from the liquid-state polarization: $P_{S S}=$ $P_{L S} \exp \left(T_{\text {trans }} / T_{1 n}\right)$. We obtained solid-state polarizations of $42.9 \pm 1.8 \%$ and $49.5 \pm 3.0 \%$ for TriPA_DNP-sample and $d_{9}$ TriPA_DNP-sample, respectively. To test the versatility and improved DNP performance of the new UV-radicals we polarized the reference substrate 1,1-bis(hydroxymethyl)cyclopropane-1${ }^{13} \mathrm{C}, \mathrm{d}_{8}$ (HP001), at the same DNP conditions of $d_{9}$-TriPA_DNPsample where $2 \mathrm{M}$ HP001 replaced the labelled glucose in the preparation. HP001 is a well suited "polarization probe" since its 
liquid state $T_{1 n}=123.0 \pm 1.0 \mathrm{~s}$. The measured ${ }^{13} \mathrm{C}$ liquid-state polarization of HP001 was $53.7 \pm 2.0 \%(n=2)$ (see supporting Information).

We compared the results achieved using UV-TriPA and UV- $d_{9-}$ TriPA to the routinely used trityl radical. The Trityl_DNP-sample was prepared by dissolving $30 \mathrm{mM}$ trityl radical $\mathrm{AH} 111501$ and 2 $M$ labelled glucose in GW55; DNP was performed at $187.94 \mathrm{GHz}$ corresponding to microwave sweep positive lobe maximum for trityl (see Supporting Information). Although $30 \mathrm{mM}$ trityl radical represents the optimal concentration to perform dDNP on [1${ }^{13} \mathrm{C}$ PA at $6.7 \mathrm{~T}$ (with $70 \%{ }^{13} \mathrm{C}$ polarization routinely obtained), ${ }^{[17]}$ Trityl_DNP-sample liquid-state polarization was not any higher than $21.1 \pm 1.5 \%(n=3)$ in good agreement with previous results. ${ }^{[8 a]}$ All relevant dDNP data are summarized in Table 1.

Table 1 The main dDNP parameters and results obtained for TriPA_DNP. sample, $d_{9}$-TriPA DNP-sample and Trityl DNP-sample at $6.7 \mathrm{~T}$ and $1.1 \mathrm{~K}$ are summarized. From the $1^{\text {st }}$ to $7^{\text {th }}$ column we report in order: sample name, radical concentration, ESR spectrum full-width at half-maximum measured at DNP conditions, solid-state buildup time constant $T_{b}$, solid-state back calculated ${ }^{13} \mathrm{C}$ polarization, liquid-state ${ }^{13} \mathrm{C}$ polarization and liquid-state relaxation time $T_{1 n}$.

\begin{tabular}{|c|c|c|c|c|c|c|}
\hline $\begin{array}{c}\text { DNP } \\
\text { Sample }\end{array}$ & $\begin{array}{c}\text { Radical } \\
\text { conc } \\
\text { [mM] }\end{array}$ & $\begin{array}{c}\text { ESR } \\
\text { linewidth } \\
{[\mathrm{MHz}]}\end{array}$ & $\begin{array}{c}S S \\
T_{b}[s]\end{array}$ & $\begin{array}{l}\text { ss } \\
\text { pol } \\
{[\%]}\end{array}$ & $\begin{array}{l}L S \\
p o l \\
{[\%]}\end{array}$ & $\begin{array}{c}L S \\
T_{1 n}[s]\end{array}$ \\
\hline TriPA & $20 \pm 1$ & 142 & $\begin{array}{c}1836 \\
\pm 28\end{array}$ & $\begin{array}{l}49.5 \\
\pm 3.0\end{array}$ & $\begin{array}{l}30.1 \\
\pm 1.8\end{array}$ & $\begin{array}{l}20.1 \\
\pm 1.0\end{array}$ \\
\hline d9-TriPA & $40 \pm 1$ & 131 & $\begin{array}{l}1230 \\
\pm 30\end{array}$ & $\begin{array}{l}42.9 \\
\pm 2.8\end{array}$ & $\begin{array}{l}26.8 \\
\pm 1.1\end{array}$ & $\begin{array}{l}19.8 \\
\pm 1.0\end{array}$ \\
\hline Trityl_DNP & 30 & 108 & $\begin{array}{c}1330 \\
\pm 28\end{array}$ & $\begin{array}{l}35.7 \\
\pm 2.5\end{array}$ & $\begin{array}{r}21.1 \\
\pm 1.5\end{array}$ & $\begin{array}{l}19.4 \\
\pm 0.8\end{array}$ \\
\hline
\end{tabular}

We verified the radicals persistency in vision of establishing a robust protocol for storage and transport of HP glucose samples. ${ }^{[7]}$ In Figure 6 we report how the UV-TriPA (panel A) and UV-d 9 -TriPA respond to temperature. In order to compensate for the Boltzmann factor and take into account the number of paramagnetic centres only, the ESR signal intensity multiplied by the temperature was plotted as a function of the latter. The characteristic "step function" profile clearly shows that all radicals are suddenly quenched above $190 \mathrm{~K}$. Thus, the samples became diamagnetic when still frozen.

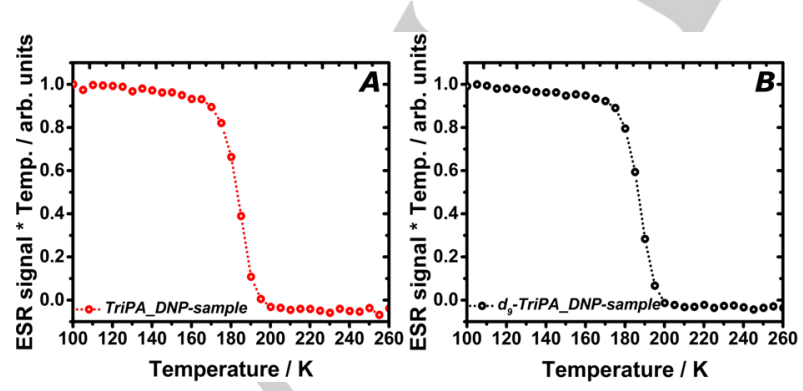

Figure 6. The ESR signal as a function of temperature is reported for TriPA DNP-sample (panel A) and $d_{9}$-TriPA DNP-sample (panel B). In both cases, the ESR signal suddenly disappeared above $190 \mathrm{~K}$. The two samples became diamagnetic when still frozen.
We finally injected $d_{9}-U V-T r i P A$ hyperpolarized $\left[U-{ }^{13} \mathrm{C}, \mathrm{d} 7\right]$ glucose into live prostate adenocarcinoma cells to judge the spectral influence of the presence of radical precursor (see Figure 7). At the current concentration the signals from the precursor are large relative to the metabolite signals in a cell experiment with limiting biological material (7 million cells). However, none of the precursor signals overlapped with metabolites from the glycolysis and had thus no influence on a kinetic analysis.

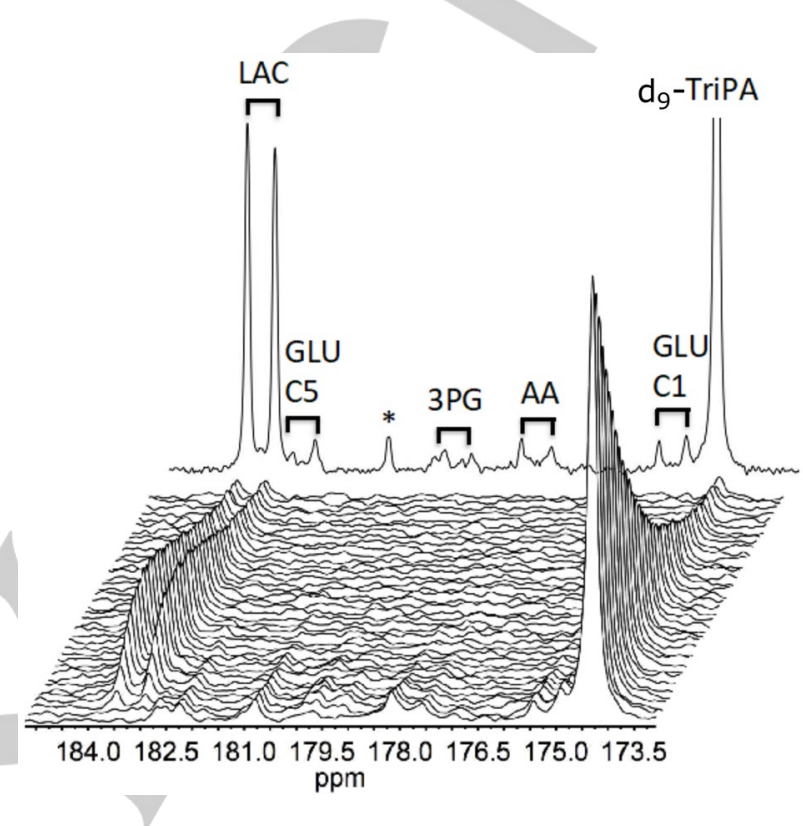

Figure 7. UV-d 9 -TriPA hyperpolarized $\left[\mathrm{U}-{ }^{13} \mathrm{C}, \mathrm{d} 7\right]$ glucose conversion in live prostate carcinoma cells (PC-3). A build-up of the glycolysis derived metabolite lactate could be followed over $30 \mathrm{~s}$. Intermediates in glycolysis are identified by ${ }^{13} \mathrm{C}-{ }^{13} \mathrm{C}$ coupling constants due to the uniformly labelled substrate and indicated by black bars in a sum of 30 spectra: Lactate (LAC), Glutamate carbon \#1 and \#5 (GLU-C1 and C5), 3-phophoglucerate (3PG), unspecified amino acid (AA). Remaining radical precursor is identified outside ppm area of interest as singlets (TriPA). The signal denoted * is likely to originate from the precursor.

The results herein show that UV-TriPA and UV-dg-TriPA, are valuable polarizing agents for ${ }^{13} \mathrm{C}$ DNP at high magnetic field. Compared to UV-PA, they benefit from a higher radical yield and improved DNP performance. These two features, respectively, are a consequence of stronger light absorption in correspondence to the $n-\pi^{*}$ electron transition and a narrower ESR spectrum due to reduced g-anisotropy and hyperfine coupling to the $\mathrm{C} 2$ position. The ${ }^{13} \mathrm{C}$ polarization level achieved was comparable to or better than trityl radical for the same sample. Moreover, their unique property of quenching above $190 \mathrm{~K}$ has two main advantages: first it allows recovering a HP solution naturally free of radical; second and more important, it represents the key feature to move towards a protocol for polarization storage. Our next aim is to decrease the radical precursor concentration to the tens of $\mathrm{mM}$ range, in order to reduce as much as possible the presence of any liquid-state background signal other than the HP substrate itself in a metabolic study. Preliminary results on samples preparation show that by doubling the UV-light power using two sources simultaneously, it is possible to achieve the same radical concentration with half of the precursor amount. As shown in Figure $3 \mathrm{~B}$, the overlap between the irradiance of the UV-source 
and the absorbance of TriPA and $d_{9}$-TriPA is relatively small. As recently demonstrated, efficient photo-generation of these labile radicals is strictly related to the photon density at the radical precursor light absorption peak. ${ }^{[18]}$

\section{Experimental Section}

Chemicals were purchased from Sigma-Aldrich, (2605 Brøndby, Denmark) excepted for the radical precursor dg-TriPA (synthesized in house, see Supporting Information) and the Trityl radical AH111501 (GE Healthcare, 01494 Amersham, UK). All experimental methods and hardware used were described previously. ${ }^{[5]}$

The UV-source (Dymax, BlueWave 75) spectral irradiance was kindly provided by the manufacturer. In the present work the UV-source was always operated at its maximum power $\left(19 \mathrm{~W} / \mathrm{cm}^{2}\right)$. Indeed, as previously demonstrated for the case of $\mathrm{PA},{ }^{[5]}$ the maximum radical yield was achieved at these experimental conditions.

It is worth pointing out that the HP sample was finally dissolved in $10 \mathrm{~mL}$ of hot $40 \mathrm{mM}$ phosphate buffer.

\section{Acknowledgements}

We tank Dr Tian Cheng for fruitful discussions. The research leading to these results has received funding from the Danish National Research Foundation (DNRF124); the European Union's Horizon 2020 research and innovation programme under the Marie Sklodowska-Curie grant agreement no. 713683 (COFUNDfellowsDTU); the Marie Skladowka-Curie ITN studentship under grant agreement No 642773 (EUROPOL); This work is part of a project that has received; the European Union's Horizon 2020 European Research Council (ERC Consolidator Grant) under grant agreement no. 682574 (ASSIMILES).

\section{Conflict of interest}

Dr. Arnaud Comment is currently employed by General Electric Medial System, Inc.

Keywords: dissolution DNP • ESR spectroscopy • hyperpolarized glucose $\cdot$ NMR spectroscopy $•$ UV-radicals

How to cite:

\section{References}

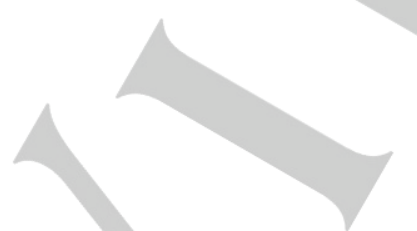

[1] a) K. Golman, R. in't Zandt, M. Thaning, P Natl Acad Sci USA 2006 103, 11270-11275; b) S. J. Nelson, J. Kurhanewicz, D. B. Vigneron,
P. E. Z. Larson, A. L. Harzstark, M. Ferrone, M. van Criekinge, J. W Chang, R. Bok, I. Park, G. Reed, L. Carvajal, E. J. Small, P. Munster V. K. Weinberg, J. H. Ardenkjaer-Larsen, A. P. Chen, R. E. Hurd, L. I. Odegardstuen, F. J. Robb, J. Tropp, J. A. Murray, Sci Trans/ Med 2013, 5, 198ra108 191-110; c) J. H. Ardenkjaer-Larsen, G. S. Boebinger, A. Comment, S. Duckett, A. S. Edison, F. Engelke, C. Griesinger, R. G. Griffin, C. Hilty, H. Maeda, G. Parigi, T. Prisner, E. Ravera, J. van Bentum, S. Vega, A. Webb, C. Luchinat, H. Schwalbe, L. Frydman, Angew Chem Int Edit 2015, 54, 9162-9185. a) J. H. Ardenkjaer-Larsen, B. Fridlund, A. Gram, G. Hansson, L. Hansson, M. H. Lerche, R. Servin, M. Thaning, K. Golman, P Natl Acad Sci USA 2003, 100, 10158-10163; b) J. H. Ardenkjaer-Larsen, J Magn Reson 2016, 264, 3-12.

[3] K. M. Brindle, J Am Chem Soc 2015, 137, 6418-6427.

[4] a) A. Capozzi, J. N. Hyacinthe, T. Cheng, T. R. Eichhorn, G. Boero, C. Roussel, J. J. van der Klink, A. Comment, J Phys Chem C 2015, 119, 22632-22639; b) T. R. Eichhorn, Y. Takado, N. Salameh, A. Capozzi, T. Cheng, J. N. Hyacinthe, M. Mishkovsky, C. Roussel, A Comment, P Natl Acad Sci USA 2013, 110, 18064-18069; c) J. A M. Bastiaansen, H. A. I. Yoshihara, A. Capozzi, J. Schwitter, R. Gruetter, M. E. Merritt, A. Comment, Magnet Reson Med 2018, 79 2451-2459.

[5] A. Capozzi, M. Karlsson, J. R. Petersen, M. H. Lerche, J. H. Ardenkjaer-Larsen, J Phys Chem C 2018, 122, 7432-7443.

a) M. L. Hirsch, N. Kalechofsky, A. Belzer, M. Rosay, J. G. Kempf, J Am Chem Soc 2015, 137, 8428-8434; b) X. Ji, A. Bornet, B. Vuichoud, J. Milani, D. Gajan, A. J. Rossini, L. Emsley, G. Bodenhausen, S. Jannin, Nature Communications 2017, 8.

A. Capozzi, T. Cheng, G. Boero, C. Roussel, A. Comment, Nat Commun 2017, 8, 15757.

8] a) M. Mishkovsky, B. Anderson, M. Karlsson, M. H. Lerche, A. D. Sherry, R. Gruetter, Z. Kovacs, A. Comment, Sci Rep-Uk 2017, 7; b) T. B. Rodrigues, E. M. Serrao, B. W. C. Kennedy, D. E. Hu, M. I. Kettunen, K. M. Brindle, Nat Med 2014, 20, 93-+; c) T. Harris, H. Degani, L. Frydman, Nmr Biomed 2013, 26, 1831-1843; d) S. Meier, M. Karlsson, P. R. Jensen, M. H. Lerche, J. O. Duus, Mol Biosyst 2011, 7, 2834-2836.

[9] a) L. Lumata, M. E. Merritt, Z. Kovacs, Phys Chem Chem Phys 2013 15, 7032-7035; b) L. Lumata, M. E. Merritt, C. R. Malloy, A. D. Sherry, Z. Kovacs, J Phys Chem A 2012, 116, 5129-5138 a) P. A. Leermakers, G. F. Vesley, J Org Chem 1963, 28, 1160-\&; b) P. A. Leermakers, G. F. Vesley, J Am Chem Soc 1963, 85, 3776\&.

[11] M. I. Guzman, A. J. Colussi, M. R. Hoffmann, J Phys Chem A 2006 110, 3619-3626.

[12] M. I. Guzman, A. J. Colussi, M. R. Hoffmann, J Phys Chem A 2006, 110, 931-935

E. C. Griffith, B. K. Carpenter, R. K. Shoemaker, V. Vaida, Proc Natl Acad Sci U S A 2013, 110, 11714-11719.

E. M. M. Weber, G. Sicoli, H. Vezin, G. Frebourg, D. Abergel, G. Bodenhausen, D. Kurzbach, Angew Chem Int Edit 2018, 57, 51715175 .

[15] J. A. Weil, J. H. Anderson, J Chem Phys 1961, 35, 1410-8

[16] a) W. T. Wenckebach, J Magn Reson 2017, 284, 104-114; b) A Bornet, J. Milani, B. Vuichoud, A. J. P. Linde, G. Bodenhausen, S. Jannin, Chem Phys Lett 2014, 602, 63-67.

[17] J. H. Ardenkjær-Larsen, S. Bowen, J. R. Petersen, O. Rybalko, M S. Vinding, M. Ullisch, N. C. Nielsen, Magnet Reson Med 2018 , accepted.

[18] I. Marco-Rius, T. Cheng, A. P. Gaunt, S. Patel, F. Kreis, A. Capozzi, A. J. Wright, K. M. Brindle, O. Ouari, A. Comment, J Am Chem Soc 2018, 140, 14455-14463. 


\section{COMMUNICATION}

Trimethylpyruvic acid is a nonexpensive and effective UVinduced radical precursor for dDNP. Its molecular structure provides high radical yield and a narrow ESR linewidth beneficial for high performance [U${ }^{13} \mathrm{C}$ ]glucose hyperpolarization

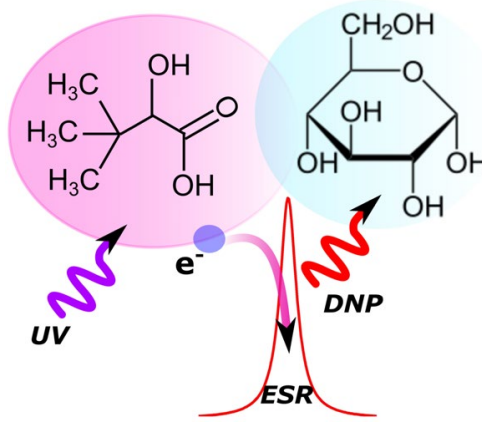

Andrea Capozzi, * Saket Patel, Christine Pepke Gunnarsson, Arnaud Comment, Mathilde H. Lerche, Magnus Karlsson, Olivier Ouari, and Jan Henrik Ardenkjær-Larsen

Page No. - Page No.

Efficient dDNP of $\mathrm{U}-{ }^{13} \mathrm{C}$-Glucose with Narrow-line UVgenerated Labile Free Radicals

${ }^{*}$ [a] Dr. A. Capozzi, Dr. M. Karlsson, MSc C. P. Gunnarsson, Dr. M. H. Lerche, Prof..J. H. Ardenkjær-Larsen

Center for Hyperpolarization in Magnetic Resonance, Department of Electrical Engineering

Technical University of Denmark, Building 349, 2800 Kgs Lyngby (Denmark)

E-mail: andcapo@electro.dtu.dk

Homepage: www.hypermag.dtu.dk

[b] MSc S. Patel, Dr. O. Ouari

Institut de Chimie Radicalire

Aix-Marseille Université, CNRS, ICR UMR 7273, 13397 Marseille Cedex 20 (France)

[c] Dr..A. Comment

Cancer Research UK Cambridge Institute

University of Cambridge, Li Ka Shing Centre, Cambridge (United Kingdom)

[d] Dr. A. Comment 